\title{
SUR L'ALEVINAGE COMBINÉ DU BLACK-BASS DU GARDON ET DE LA CARPE
}

\author{
Par A. WURTZ \\ Chef de Travaux des Eaux et Forêts \\ Chargé de la Station d'Hydrobiologie appliquée du Paraclet.
}

\begin{abstract}
Beaucoup de choses ont déjà été écrites sur l'alevinage des Carpes, des Gardons ou des Black-bass, et, au premier abord, en ce qui concerne la pisciculture particulière de chacune de ces espèces isolées (élevage non combiné), il semblerait même qu'il ne reste plus rien à dire. Cependant, lorsque l'on pratique l'élevage de ces poissons en vue d'une production quelque peu importante d'alevins de repeuplement, on est toujours exposé à de bonnes ou à de mauvaises surprises. Cela prouve que le pisciculteur ne "possède " pas encore assez la technique de l'alevinage pour pouvoir prévoir à coup sûr qu'il aura tant d'alevins de Carpes, de Gardons ou de Black-bass. Cela prouve également qu'il reste encore beaucoup de détails biologiques à mettre au point.
\end{abstract}

En Salmoniculture, où l'on opère par fécondation artificielle, on peut prévoir à peu près le nombre d'alevins que l'on produira, à condition, toutefois, que les conditions physiques (température de l'eau, oxygène, etc.) soient bonnes et qu'il n'y ait pas de maladies. En Cypriniculture la méthode de ponte des Carpes en frayères Dubisch, et l'élevage des alevins en bassins de grossissement permettent, dans une certaine mesure, d'avoir des résultats certains, bien qu'il y ait encore de nombreuses difficultés techniques et que les conditions atmosphériques interviennent en pouvant faire échouer, ou échelonner la ponte.

En ce qui concerne la production intensive de Gardons, de Black-bass (et aussi de Tanches d'ailleurs), on est plus ou moins livré au hasard d'une bonne ou d'une mauvaise ponte : on met les géniteurs en étang, et l'on attend...

Le but de cet article n'est pas d'apporter une solution définitive au problème de la production à volonté d'alevins de Carpes, de Gardons ou de Black-bass; je voudrais simplement relater quelques essais entrepris dans les étangs de la Station d'Hydrobiologie appliquée du Paraclet, pour montrer jusqu'à quel point l'alevinage combiné de la Carpe, du Black-bass et du Gardon est possible.

J'avais eu l'occasion de constater qu'en étang clos, le Black-bass, 
loin d'être un vorace destructeur, pouvait jouer le rôle de calibreur d'alevins de Poissons blancs. Cette remarque avait été faite lors de la pêche de l'étang $E^{\theta}$ du Paraclet (d'une superficie de 2 hectares) au printemps 1950.

L'empoissonnement de cet étang, au printemps 1949 (Avril) avait été le suivant :

45 géniteurs Gardons et 60 géniteurs Rotengles;

20 géniteurs Brèmes;

50 géniteurs Tanches;

20 Carpes de 2 à 3 étés (dont on savait pas très bien si elles étaient des géniteurs ou non);

6 géniteurs Black-bass.

Cet empoissonnement, que je ne contrôlais pas à l'époque, était criticable; on comprend facilement que ce fût une erreur d'introduire dans un mẻme étang les trois espèces de Poissons blancs (Gardons, Rotengles, Brèmes) susceptibles de s'hybrider. D'autre part, l'empoissonnement en géniteurs Black-bass semblait trop faible pour deux hectares.

Les résultats de la pêche au mois de Mars 1950 (après 11 mois) furent assez surprenants :

1.700 alevins de Gardons de 10 à 12 centimètres (poids moyen 40 gr.), dans la mesure où il a été possible de les reconnaître, en raison de leur hybridation avec les Rotengles;

2.800 alevins de Rotengles de 8 à 10 centimètres (poids moyen 15 gr.) ;

1.500 alevins de Brèmes, longs de 15 à 20 centimètres, pesant en moyenne 75 grammes, soit une croissance excellente, représentant un poids de $112 \mathrm{~kg}$. d'alevins de Brèmes pour les deux hectares d'étang. (Il faut dire que les étangs et tourbières de la Somme conviennent bien à la Brème, et que celle-ci, bien que peu intéressante, $y$ pousse remarquablement). On a trouvé dans cet étang quelques gros alevins hybrides de Gardons et Brèmes (Brème de Buggenhagen);

65 Carpes de 400 grammes environ; ce sont des alevins qui, s'étant maintenu en petit nombre dans l'étang, ont également grossi d'une manière surprenante ;

700 alevins de Tanches de 15 à 20 centimètres;

550 alevins de Black-bass de 8 à 12 centimètres (15 à 40 gr.).

On a retrouvé les géniteurs qui avaient été mis dans l'étang, et en plus quelques Goujons et Vandoises, parvenus sans doute dans l'étang, à l'état de tout jeunes alevins par un tuyau d'alimentation communiquant avec la rivière. Bref une productivité nette, assez honnête de $280 \mathrm{~kg}$. d'alevins, soit $140 \mathrm{~kg}$. à l'hectare.

Mais le résultat le plus remarquable de cette pêche a été le fait qu'il n'y avait pas un seul alevin de Poisson blanc d'une taille inférieure à 8 centimètres. Abstraction faite des Rotengles, dont la pisciculture n'est pas à conseiller, la plupart des alevins de l'année avaient plus de 12 centimètres. L'uniformité de la dimension des alevins était étonnante. 
Il y avait tout lieu de supposer que les Black-bass étaient les responsables de ce calibrage.

Essais montrant le role du Black-bass élevé avec des Carpes.

Les essais de 1950 ont eu pour but de vérifier le rôle calibreur du Black-bass, en présence d'alevins de Carpes. Deux étangs, appelés $\mathrm{E}^{7}$ et $E^{8}$, ont été consacrés à ces essais. Primitivement, chacun de ces étangs devait avoir une surface de 90 ares, mais par suite d'un mauvais nivellement, ils ne se sont remplis que partiellement : la surface en eau de l'étang $E^{7}$ était de 40 ares seulement, celle de l'étang $E^{8}$ était de 30 ares. La pente était cependant régulière, et la plus grande profondeur à la pêcherie était d'environ 1 mètre.

Empoissonnement fin Avril 1950. — L'étang $\mathrm{E}^{7}$ a reçu :

200 Gardons, dont environ une centaine de géniteurs de plus de 15 centimètres;

10 géniteurs Carpes;

8 géniteurs Black-bass;

25 alevins de Black-bass de 8 à 12 centimètres.

L'ètang $\mathrm{E}^{8}$ a reçu :

125 géniteurs Gardons;

50 géniteurs Tanches;

8 géniteurs Carpes.

Je reconnais volontiers que cet empoissonnement a été un peu fort, car j'avais calculé avec une surface d'eau d'environ 1 hectare pour chacun des étangs. On a seulement constaté, après la mise en eau, que les étangs se remplissaient partiellement et donnaient une surface d'eau utilisable de 30 ares et 40 ares respectivement. On verra d'ailleurs à la pêche que ces chiffres au fond n'étaient pas trop forts et que les étangs n'étaient nullement surpeuplés.

Comme ces étangs étaient en eau pour la première fois, il n'a pas paru utile d'y introduire des engrais. Ils ont été simplement faucardés une seule fois en juillet, pour sortir les roseaux qui avaient tendance à se développer trop abondamment. La queue des étangs, remontant en pente douce, réalisait à peu près toutes les conditions d'une bonne frayère. Je ne fondais cependant pas grand espoir sur ces étangs : les récoltes de plancton faites au cours du printemps et de l'été paraissaient pauvres : quelques Algues filamenteuses, des Diatomées, et, comme zooplancton, des Copépodes. La pêche devait être contraire aux prévisions et montrait l'intérêt de l'expérience dans deux étangs neufs.

Pêche. - Les deux étangs $\mathrm{E}^{7}$ et $\mathrm{E}^{8}$ ont été vidés le 9 Octobre 1950. L'étang $\mathrm{E}^{7}$ (40 ares) a donné les résultats suivants :

150 géniteurs Gardons environ retrouvés, les autres morts ou disparus.

Pas d'alevins de Gardons!

10 géniteurs Carpes retrouvés, grossis. 
6.j00 alevins de Carpes de 6 à 10 centimètres, quelques-uns même de 10 à 12 centimètres, pesant 8 gr. j̀ à 20 gr. 5 (poids moyen : 13 gr.), soit un poids total de $84 \mathrm{~kg}$ d'alevins de Carpes.

15 gros géniteurs Black-bass retrouvés, grossis, certains pesant jusqu’à 700 grammes.

4.500 alevins de Black-bass, dont 4.000 de 6 à 8 centimètres et 500 de 9 à 14 centimètres, pesant de 13 à 25 grammes, soit un poids total de $33 \mathrm{~kg}$ d'alevins de Black-bass.

Sans compter le gain en poids des géniteurs, que je me permets de négliger pour mieux faire ressortir les chiffres d'alevinage, cela fait $117 \mathrm{~kg}$ d'alevins (Carpes et Black-bass) pour 40 ares, soit une productivité nette, fort honnête, de $292 \mathrm{~kg}$ à l'hectare.

L'étang $\mathrm{E}^{8}$ (30 ares) a donné les résultats suivants :

Environ 70 géniteurs Gardons ont été retrouvés.

45.000 alevins de Gardons de 5 à 8 centimètres, pesant en moyenne 2, 3 grammes, soit un poids total de $110 \mathrm{~kg}$ d'alevins de Gardons.

Les 8 géniteurs Carpes ont été retrouvés, mais aucun alevin de Carpe! Les géniteurs Tanches ont également été retrouvés, mais pas d'alevins de Tanche!

Il convient de signaler une vingtaine de Perches de 12 à 15 centimètres, venues à l'état de petits alevins par la rivière, et qui ont grossi dans l'étang.

Cela nous donne pour cet étang, toujours sans compter les adultes, $110 \mathrm{~kg}$ pour 30 ares, soit une productivité nette de $360 \mathrm{~kg}$ à l'hectare.

Les enseignements à tirer de l'alevinage dans ces deux étangs sont des plus intéressants.

Au point de vue quantitatif d'abord, on peut dire que la productivité des deux étangs est bonne, puisqu'il a été retiré rien qu'en alevins, pour une superficie totale de 70 ares, $227 \mathrm{~kg}$ de poissons, ce qui donne une productivité de $324 \mathrm{~kg}$ à l'hectare.

Mais si l'on tient compte de la production qualitative, et surtout de la production combinée d'alevins de Gardons, de Carpes et de Black-bass, qui était en principe le but de l'expérience, il se dégage un certain nombre de constatations, que je vais résumer en trois points.

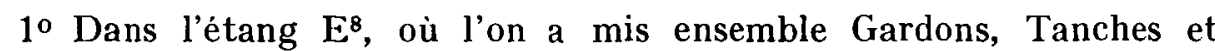
Carpes, seuls les Gardons se sont reproduits. J'avoue ignorer complètement pourquoi les Carpes ne se sont pas reproduites, car les conditions étaient bonnes et paraissaient identiques à celles de l'étang $E^{7}$. A plus forte raison peut-on etre surpris de ce que la reproduction des Tanches n'a pas réussi non plus, car généralement l'alevinage des Tanches réussit assez bien au Paraclet. A titre d'exemple, il suffirait de citer le cas d'un petit bassin de 5 ares, où il a été introduit au printemps 1950 des géniteurs hybrides Gardons $\times$ Rotengles, dans le but de séparer les espèces à la première génération. A la pêche, en automne, on a retiré en plus de 
3.000 alevins de Gardons $\times$ Rotengles hybrides (1), 1.000 alevins de Tanches de 8 à 10 centimètres provenant de 3 géniteurs qui étaient restés envasés. En ce qui concerne l'alevinage combiné de Tanches et de Gardons, à la lumière des essais entrepris, et pour des raisons qui n'entrent pas dans le cadre de cet article, je crois qu'il vaut mieux élever les Tanches et les Gardons dans des étangs séparés. Dans l'étant $\mathrm{E}^{8}$ le nombre d'alevins de Gardons produits (45.000 pour 30 ares, soit 150.000 à l'hectare) est satisfaisant, mais les alevins de 5 à 8 centimètres sont bien petits, fragiles pour le transport et ne me paraissent pas avoir la taille optimale pour le réempoissonnement.

$2^{0}$ Dans l'étang $E^{7}$, avec Gardons, Carpes et Black-bass, l'alevinage combiné des Carpes et des Black-bass a parfaitement réussi. La reproduction de la Carpe n'a rien de particulièrement surprenant car elle a réussi un peu partout en France, sans que l'on sache d'ailleurs à quel ensemble de conditions est due la réussite (l'influence de la température et le réchauffement de l'eau seuls ne suffisent pas). Il y a des années favorables à la reproduction de la Carpe, comme il y a des années de bon vin...

Ce qui est plus surprenant, c'est que dans cet étang, les Gardons se sont reproduits, car j'ai vu de nombreux alevins au début de l'été. Or il n'en a pas été retrouvé un seul à la pêche. J'ai la certitude que les Black-bass ont " nettoyé " complètement les alevins de Gardons et s'en sont nourris sélectivement jusqu'au dernier, tout en respectant les Carpes. Cette prédilection des Black-bass pour le menu fretin que représentent les alevins de Gardons, s'explique sans doute par la différence de croissance des trois sortes d'alevins en présence. Lorsque les jeunes alevins de Black-bass, cessant d'être planctophages pour devenir ichtyophages, se mettent à chasser les autres alevins, ceux de Carpes (qui étaient des alevins de Carpes sans écailles, sélectionnées, à croissance rapide) étaient déjà trop gros pour pouvoir être mangés. Ce sont les petits Gardons qui représentaient les proies les plus faciles.

On peut donc en conclure que dans les petits étangs, l'alevinage combiné de la Carpe et du Black-bass réussit fort bien, à condition de donner à ce dernier des alevins de Gardons, dont il semble démontré qu'il fait sa nourriture préférentielle.

Ce fait nous permettra d'ailleurs de constater que le Black-bass n'est pas aussi vorace qu'on a voulu le faire croire. N'oublions pas qu'il a été retiré de l'étang 15 gros Black-bass de 700 grammes. Si en plus de cela, dans une surface d'eau aussi restreinte, on retire 4.500 alevins de Blackbass et 6.500 alevins de Carpes, il faut convenir que les Black-bass n'ont pas tout mangé.

(1) A signaler à ce sujet : $1^{0}$ que les Gardons et les Rotengles s'hybrident, ce qu i n'est pas nouveau; $2^{\circ}$ que ces hybrides sont féconds et donnent des produits hybrides présentant les caractéres intermédiaires entre les Gardons et les Rotengles ; $3^{\circ}$ qu'il est très difficile après l'hybridation de redégager une lignée spéciflquement pure; $4^{\circ}$ que le caractère "Rotengle " paraît dominant. 
On pourrait discuter longuement sur les avantages et les inconvénients de ces procédés de nutrition. Il y a ceux qui aiment les Gardons, et il y a ceux qui n'aiment pas les Black-bass. Au point de vue économie piscicole, je pense toutefois que tout le monde sera d'accord pour reconnaître qu'il est intéressant de transformer de la chair de Gardon, médiocre, en chair de Black-bass, excellente, de l'avis de tous ceux du moins qui $y$ ont goûté.

$3^{\circ}$ Pour illustrer l'intérêt de l'expérience d'alevinage combiné, il est bon d'aligner encore quelques chiffres :

L'étang $\mathrm{E}^{\mathbf{8}}$ a produit 40.000 alevins de Gardons vendables (le chiffre réel est baissé de $10 \%$ pour tenir compte de la mortalité au moment des manipulations). En nous reportant aux prix fixés par le Ministère de l'Agriculture (1.000 fr. le mille en 1951), l'étang aura produit 40.000 francs d'alevins.

L'étang $\mathrm{E}^{7}$ a produit (et le chiffre réel sera également baissé de $10 \%$ )

- 6.000 alevins de Carpes, vendables au prix officiel de $7 \mathrm{fr} .50$ pièce en moyenne, soit 45.000 francs de Carpes,

- et 4.000 alevins de Black-bass valant 9 fr. 50 pièce, soit 38.000 francs de Black-bass ; ce qui fait en tout 83.000 francs d'alevins pour l'étang $E^{7}$ (valeur en 1951).

Ces chiffres sont assez éloquents pour parler en faveur de l'étang $E^{7}$ et de l'alevinage combiné Carpes-Black-bass, en général.

\section{Role du Black en élevage combiné Carpes-Black-bass EN Étangs plus grands.}

Au cours de l'essai relaté au début de cet article, on a déjà pu se rendre compte que dans un étang de plus d'un hectare on pouvait produire à la fois des alevins de Gardons (ou Rotengles, ou Brèmes) bien calibrés, des Black-bass, et le cas échéant des Carpes ou des Tanches. L'empoissonnement ayant été assez mal réalisé, les conclusions n'avaient qu'un rôle indicatif. Voici les résultats de la pêche du même étang $\mathrm{E}^{9}$ au printemps 1951, l'empoissonnement ayant été fait d'une manière plus rationnelle :

Empoissonnement au printemps (Avril) 1950. - Il a été mis dans l'étang $\mathrm{E}^{9}$ de 2 hectares :

200 géniteurs Gardons ;

55 Carpes, dont 18 de 400 grammes, et 37 alevins de 10 à 12 centimètres ;

50 géniteurs Tanches;

12 géniteurs Black-bass.

Résultats de la pêche au printemps (Mars) 1951 :

9.000 alevins de Gardons, exactement calibrés à 12 centimètres, poids moyen : 25 grammes;

48 Carpes d'environ $1 \mathrm{~kg}$ (à remarquer la croissance surprenante 
des alevins de 10 à 12 centimètres, en raison du petit nombre de Carpes dans l'étang);

2.000 alevins de Tanches de 8 à 10 centimètres;

4.500 alevins de Black-bass de 13 à 14 centimètres, quelques-uns jusqu'à 18 centimètres, soit $120 \mathrm{~kg}$ d'alevins de Black-bass.

La productivité totale en alevins s'élève à $405 \mathrm{~kg}$. pour les 2 hectares, ce qui semble correspondre à peu près au maximum à atteindre dans les étangs-tourbières de la Somme.

Le Black-bass est loin d'avoir nui à la population piscicole de l'étang : au contraire, celle-ci semble mieux équilibrée. On a produit des alevins de Black-bass, de Gardons et de Tanches (ou de Carpes, le cas échéant), espèces pouvant donc vivre en parfaite harmonie.

Il se confirme que dans les étangs d'une surface égale ou supérieure à un hectare, le Black-bass joue le rôle de calibreur d'alevins, en détruisant le menu fretin, qui de toute manière serait inutilisable. Ainsi que l'ont montré les divers essais entrepris au Paraclet, on peut s'attendre à produire, en compagnie du Black-bass, des alevins de Gardons et de Carpes en quantités peut-être légèrement moindres, mais beaucoup plus gros, uniformément calibrés, ayant donc une valeur beaucoup plus grande comme alevins destinés au repeuplement. 